\title{
Descubre tu Potencial para Educar a tus Hijas e Hijos: un programa de parentalidad positiva
}

\author{
Enrique B. Arranz-Freijo \\ Universidad del País Vasco/Euskal Herriko Unibertsitatea (UPV/EHU) \\ e.arranzfreijo@ehu.eus
}

Florencia Barreto-Zarza

Universidad del País Vasco/Euskal Herriko Unibertsitatea (UPV/EHU)

\section{Loli García-García}

Servicio de Infancia y Familia, Ayuntamiento de Vitoria-Gasteiz

\section{Silvia Sánchez-Pinedo}

Servicio de Infancia y Familia, Ayuntamiento de Vitoria-Gasteiz

\author{
Aitziber Martínez de la Hidalga \\ Servicio de Infancia y Familia, Ayuntamiento de Vitoria-Gasteiz
}

\begin{abstract}
Lan honetan, "Ezagutu zure seme-alabak hezteko ahalmena” programa aurkezten da. Programa honen bidez, familia-testuinguruaren kalitatearen ebaluazioa egiten da, eta, horretarako, 0-3 urte bitarteko haurrak dituzten familiei bisita egiten zaie etxean, hezkuntza, gizarte eta osasun esparruetatik abiatuta. Ebaluazioaren ondoren esku-hartze fase bat eta esku-hartze horren eraginaren ebaluazio bat egingo da. Emaitzek erakusten dutenez, gurasoen gaitasunei buruzko esku-hartze psikohezitzaileak, hala nola jolasa sustatzea, garapen kognitiboa sustatzea, estresari aurre egitea eta beste batzuk, hobetu egiten du familien jarduera gaitasun horietan. Programa ezartzeko prozesuan, hobetu beharreko arloak aurkitzen dira, eta gurasotasun positiboaren gakoetan oinarritutako ekintza politikora bideratuko dira honen inplikazioak.
\end{abstract}

\section{GAKO-HITZAK:}

Familia, lehen haurtzaroa, familiako esku-hartzea, ahaidetasun positiboa, ebaluazio-programak, prebentzio-programak.
En este trabajo se presenta el programa Descubre tu Potencial para Educar a tus Hijos e Hijas, que consiste en una evaluación de la calidad del contexto familiar, realizada por medio de una visita domiciliaria a familias con niños y niñas entre cero y tres años derivadas desde los ámbitos educativo, social y sanitario. La evaluación es seguida por una fase de intervención y de una evaluación del impacto de dicha intervención. Los resultados muestran que la intervención psicoeducativa sobre competencias parentales tales como la promoción del juego, la promoción del desarrollo cognitivo, el afrontamiento adaptativo del estrés y otras, mejora el desempeño de las familias en tales competencias. Durante el proceso de implementación del programa, se detectan áreas de mejora y se derivan implicaciones para la acción política basada en las claves de la parentalidad positiva.

\section{Palabras Clave:}

Familia, primera infancia, intervención familiar, parentalidad positiva, programas de evaluación, programas de prevención. 


\section{Introducción}

El programa Descubre tu Potencial para Educar a tus Hijos e Hijas se enmarca en el desarrollo del II Plan de Infancia y Adolescencia (PLINA) del Ayuntamiento de Vitoria-Gasteiz, diseñado por el Servicio de Infancia y Familia perteneciente al Departamento de Políticas Sociales y Salud Pública. En el tercer objetivo de la primera línea estratégica del PLINA, aprobado en mayo de 2018 , se señala la necesidad de promover el ejercicio de la parentalidad positiva por parte de madres y padres. Como desarrollo de este objetivo, el Servicio de Infancia y Familia en colaboración con el equipo de investigación Haezi-Etxadi de la Universidad del País Vasco/Euskal Herriko Unibertsitatea (UPV/ EHU), ha elaborado un Programa de Parentalidad Positiva (Servicio de Infancia y Familia y Haezi-Etxadi, 2019) para ser aplicado en el periodo comprendido entre los años 2018-2022 y que fue aprobado el 11 de enero de 2019.

El programa tiene una vocación transversal interdepartamental y en sus líneas articula medidas de promoción de la parentalidad positiva en los diferentes departamentos del Ayuntamiento. La segunda línea estratégica del citado programa tiene como objetivo la formación de padres, madres y personas cuidadoras en las competencias propias de la parentalidad positiva, y en la sublínea 2.1 se explicita la necesidad de activar acciones universales de promoción de la parentalidad positiva dirigidas a los colectivos mencionados. En este contexto, el programa que se presenta en este artículo constituye un desarrollo de esa sublínea.

Como cobertura a la acción municipal en la Comunidad Autónoma de Euskadi (CAE), se debe mencionar el IV Plan Interinstitucional de Apoyo a las Familias de la CAE 2018-2022 (Gobierno Vasco, 2018) elaborado por el Gobierno Vasco (Dirección de Política Familiar y Diversidad/Consejería de Asuntos Sociales). El citado plan recoge medidas incluidas en el informe previo Estudio de campo de los programas y actividades de parentalidad positiva existentes en la Comunidad Autónoma del País Vasco (Gobierno Vasco, 2012; Arranz et al., 2016a), elaborado por el grupo de investigación Haezi-Etxadi de la UPV/EHU para la Dirección de Política Familiar y Comunitaria del Departamento de Empleo y Asuntos Sociales del Gobierno Vasco. Asimismo, el citado plan interinstitucional se ubica en el marco conceptual del Pacto Vasco por las Familias y la Infancia; uno de los compromisos que formula se refiere directamente a la parentalidad positiva. En él se recomienda reforzar los programas de parentalidad positiva, de salud infantil, de empoderamiento de las mujeres, de prevención de conflictos y de mediación familiar, de cara a reforzar las capacidades parentales y educativas de padres y madres.

Por otra parte, el eje 2 de los objetivos estratégicos formulados en el plan se refiere también a las acciones de parentalidad positiva ligadas al fomento en la población de madres, padres y cuidadores de competencias de promoción del apego seguro y, a la vez, proveer a las mismas de apoyo especializado en las situaciones de dificultad para llevar a cabo una crianza adecuada de sus hijas e hijos. En los objetivos 4, 15, 16 y 18 del citado plan interinstitucional se incluyen diversas actuaciones relativas a la parentalidad positiva. En particular, la medida 103 del objetivo 16 propone la realización de campañas de sensibilización relativas a la promoción de la adquisición, por parte de las familias, de competencias parentales. La filosofía de fondo de las medidas expuestas consiste en la implementación de políticas de prevención primaria dirigidas al mayor número posible de familias.

Los antecedentes empíricos del programa que se presenta en este documento parten del informe técnico Análisis de los programas y actividades de parentalidad positiva en el Ayuntamiento de Vitoria-Gasteiz (2015), realizado por el equipo de investigación Haezi-Etxadi de la UPV/EHU, en colaboración con el Servicio de Infancia y Familia del Ayuntamiento de Vitoria-Gasteiz. Entre las conclusiones del informe se destaca la ausencia de programas sistemáticos de parentalidad positiva en el tramo de edad desde cero a tres años de la población infantil. Igualmente, se indicó la necesidad, basada en la revisión de la literatura científica, de desarrollar intervenciones preventivas sobre la promoción del vínculo prenatal. Otro precedente empírico de la propuesta de este programa, viene constituida por el estudio piloto del propio programa, cuyos resultados se encuentran publicados en revistas nacionales e internacionales (Arranz-Freijo et al., 2016b, 2019) en el que se aportan datos, obtenidos sobre una población de cincuenta familias, que apoyan el impacto positivo de un programa de evaluación de la calidad de los contextos familiares de las familias participantes. Las propuestas de mejora que se derivan de los resultados del mencionado estudio piloto, han sido incorporadas en su totalidad en la realización de la nueva propuesta del programa Descubre tu Potencial para Educar a tus Hijos e Hijas.

La propuesta del programa alcanza mayor dimensión si se tiene en cuenta que va a constituir el precedente del programa Senidegune, implementado ya de manera estable por el Servicio de Infancia y Familia del Ayuntamiento de Vitoria-Gasteiz y dirigido a familias con niños y niñas a partir de los tres años. Este programa se entronca igualmente en el ámbito de las políticas preventivas de empoderamiento familiar con el enfoque de la parentalidad positiva, basadas en la evidencia científica (Arranz y Rodrigo, 2018). Con la estabilización de la oferta de dos programas consecutivos se consolidaría una acción preventiva de alta calidad a disposición de las familias residentes en el municipio de Vitoria-Gasteiz.

\subsection{Fundamentos científicos}

Este programa de intervención familiar tiene su origen en el aumento del conocimiento empírico 
de los últimos años en las áreas de psicología evolutiva y psicología de la familia. La confluencia de ambas áreas ha conducido al desarrollo de la línea de intervención con familias denominada parentalidad positiva, que se encuentra claramente apoyada por diversas Administraciones Públicas (Rec. 19 del Consejo de Ministros de Europa, 2006). Los fundamentos básicos del programa descrito en este artículo proceden del modelo teórico de las Dimensiones de la Parentalidad y del Currículum Óptimo de Parentalidad Positiva (COPP) (Arranz et al., 2016b; Arranz y Rodrigo, 2018). Las competencias parentales recogidas en el COPP proceden de variables identificadas por la investigación reciente como influyentes en el desarrollo infantil y susceptibles de intervención a nivel de fortalecimiento o psicoeducación parental.

Dentro de sus principios fundamentales, el enfoque de la parentalidad positiva contempla que la crianza en la sociedad actual plantea un importante desafío para madres y padres. Por tanto, la promoción del desarrollo infantil requiere de una relación afectiva y sostenedora, un contexto familiar estructurado y sostenible, y adaptado a las etapas evolutivas infantiles. Asimismo debe tenerse en cuenta que la inversión en la crianza es un capital social donde la familia extensa, la red social, formal e informal, y la comunidad desempeñan un papel destacado (Haezi-Etxadi, 2017; Rodrigo et al., 2015). La perspectiva de la parentalidad positiva señala igualmente que la protección del bienestar infantil pasa por la necesidad de inversión en programas, proyectos y servicios orientados a fomentar el cuidado competente y los buenos tratos en el sistema familiar. Estas propuestas pueden partir de diferentes ámbitos, bien sea el sanitario, el educativo, o el social, o contemplar una perspectiva multidisciplinar (Arranz y Rodrigo, 2018; HaeziEtxadi, 2017; Rodrigo et al., 2015).

El planteamiento de la parentalidad positiva tiene importantes implicaciones teóricas y metodológicas. A nivel teórico se asume la complejidad del proceso de crianza familiar, entendiendo que este es un proceso influenciado por múltiples factores que son susceptibles de ser medidos, incluso antes del nacimiento, y que pueden ser igualmente objeto de intervenciones preventivas y de promoción. A nivel metodológico, la apuesta por la promoción del desarrollo psicológico saludable, en lugar del tratamiento o la prevención, plantea un cambio de perspectiva tanto en la visión de la población que accede a la intervención como en el diseño y parámetros de esta.

Tal y como expone la Organización Mundial de la Salud (2004), la promoción debe contemplarse como algo más que la ausencia de problemáticas o la evitación de la aparición de las mismas. Desde este punto de vista, el enfoque salutogénico es una herramienta teórica eficaz. Dicho enfoque parte de que las intervenciones deben orientarse a identificar los recursos (endógenos, exógenos, formales e informales), las habilidades y los intereses de las familias, orientados al mantenimiento, $y$ al fomento de su bienestar (Roncallo, Barreto y Sánchez de Miguel, 2018). Esta propuesta sugiere que los programas de intervención deben tener un diseño dinámico que permita identificar los activos y las prioridades de los participantes, proporcionando además el apoyo necesario para la movilización de lo ya existente y el aprendizaje de nuevas herramientas (Juvinya-Canal, 2013; Morgan y Hernán, 2013). Otro punto interesante es que el enfoque salutogénico asume que el bienestar no es un proceso estático. De esta manera, los protocolos de los programas deben adecuarse a las diferentes etapas y necesidades de la población diana.

En el contexto de la parentalidad positiva, el trabajo desde la promoción implica el fomento del bienestar del sistema familiar y, a su vez, la estimulación del óptimo desarrollo infantil. Por tanto, la salutogénesis buscaría potenciar los recursos de los sistemas familiares, así como generar una positiva percepción de su estado, su capacidad de autorregulación y de regulación del entorno (Pérez-Botella, Downe, Magistretti, Lindstrom y Berg, 2015). Todos los objetivos planteados se formulan entendiendo que las necesidades de parentalidad obedecen a procesos adaptativos, etapas del desarrollo infantil $y$ contextos sociales cambiantes, los cuales plantean diferentes procesos, retos y oportunidades. De esta forma y recogiendo la visión conjunta de diferentes profesionales expertos a nivel estatal (Arranz y Rodrigo, 2018; Haezi-Etaxdi, 2017; Rodrigo et al., 2015), se plantea que las políticas familiares que apoyen la implementación de programas y proyectos con esta orientación deben poseer las siguientes características:

- De enfoque preferentemente universal: se busca un acceso de las familias de forma libre, voluntaria y no estigmatizada. Dirigidas a la población general y no solo a poblaciones de riesgo.

- Centradas en la promoción: orientadas a la adquisición y promoción de competencias parentales que fomenten el bienestar familiar y el desarrollo infantil integral. Esto supone la utilización de un enfoque salutogénico, no basado en criterios de déficit o psicopatología.

- De acercamiento grupal, individual o mixto a cada familia y basadas en la metodología experiencial constructivista que implica la reflexión conjunta, el aprendizaje significativo y la creación de redes sociales de crianza.

- Respetuosas con la libertad de las familias: con un enfoque de la crianza como un proceso de toma de decisiones libres y basadas en conocimiento científico contrastado.

- Basadas en un modelo de intervención contextualizado en la realidad de cada una de las familias participantes.

- Implementadas por profesionales especializados con conocimiento y experiencia en el área 
de psicología de la familia y con un enfoque interdisciplinar.

- Fundamentadas, preferentemente, en un planteamiento de I+D+I (investigación-desarrolloinnovación), que incorpore los resultados de la implementación de los programas en la mejora constante de estos.

- Apoyadas en un enfoque basado en la evidencia: receptoras de transferencia de conocimiento científico en el diseño teórico y metodológico de los programas.

La propuesta de competencias parentales que serán promocionadas en el programa Descubre tu Potencial para Educar a tus Hijos e Hijas sigue el esquema teórico de las Dimensiones de la Parentalidad, que constituye igualmente el modelo teórico del Programa de Parentalidad Positiva del Ayuntamiento de Vitoria-Gasteiz. Siguiendo el esquema presentado en publicaciones anteriores (Arranz-Freijo et al., 2016b, 2019) se expone a continuación un resumen del mencionado esquema teórico:

La primera dimensión es denominada buen trato: el concepto de buen trato, entendido de forma mucho más completa y compleja que la mera ausencia de maltrato, se refiere a la existencia en el contexto familiar de condiciones que conduzcan a la cristalización de vínculos de apego seguro con la madre, el padre y/u otros cuidadores. El complemento necesario a las vinculaciones seguras está constituido por la práctica parental de la frustración óptima que va a conducir a una adecuada gestión de la expresividad y regulación emocional y al desarrollo de la autonomía, la autoestima y la resiliencia (Anaut y Cyrulnik, 2014; Barudy y Dantagan, 2005; DeHart, Pelham, y Tennen, 2006). La frustración óptima (Pesic y Baucal 1996; Arranz, 2002) es definida como la experimentación, por parte de niños y niñas, de frustraciones adecuadamente temporalizadas, dosificadas y nunca traumáticas, que ofrecen retos accesibles que activan la construcción de respuestas adaptativas exitosas a su entorno. La metáfora de la vacuna psicológica aporta una mayor clarificación conceptual de esta práctica parental de carácter transversal.

La segunda dimensión es la promoción del desarrollo: esta dimensión incluye el potencial de estimulación del desarrollo que se encuentra en un contexto familiar y se concreta en la presencia en el hogar de materiales de estimulación del aprendizaje, en la práctica del juego en familia (Milteer, Gisnburg Council on Communications and Media Committee on Psychosocial Aspects of Child and Family Health y Mulligan, 2012), en la presencia de promoción apropiada del desarrollo cognitivo y lingüístico (LugoGil y Tamis-Lemonda, 2008), también en la diversidad de experiencias que la familia ofrece a los niños y niñas y en la calidad del entorno físico (Galende, Sánchez de Miguel y Arranz, 2011) en el que convive cada unidad familiar. El complemento a la promoción del desarrollo se halla en el denominado perfil parental potenciador, dentro del cual emerge como una variable esencial la autoeficacia parental, definida como la percepción que madres, padres o personas responsables del niño/a poseen sobre sus propias competencias para llevar a cabo el proceso de crianza de manera saludable; la investigación reciente muestra el impacto positivo de un alto nivel de autoeficacia parental posee sobre el desarrollo infantil (Shumow y Lomax, 2002; Fang et al., 2021). La intervención para la promoción de la variable autoeficacia parental se ha convertido en un objetivo fundamental de la intervención psicoeducativa a través de programas de parentalidad positiva (Arranz-Freijo y Barreto-Zarza, 2021).

La tercera dimensión es denominada ecología potenciadora de la parentalidad e incluye el apoyo social que dispone una familia en sus tareas de crianza, que se concreta en factores como la implicación del padre (Huerta et al., 2014), denominada actualmente como corresponsabilidad parental (Lidbeck y Boström, 2021), así como la calidad de las relaciones con la familia extensa (Jaeger, 2012) y la calidad de las relaciones entre la familia y la escuela (Crosnoe, 2015). La ecología familiar está también constituida por la tensión existente en el sistema familiar, que se manifiesta a través de los niveles de conflicto y estrés que viven las familias y que, cuando alcanzan niveles excesivos, no facilitan la práctica de una parentalidad positiva (Bloomfield y Kendall, 2012; Hanington, Heron, Stein y Ramchandani, 2012). En el modelo actualizado de las dimensiones de la parentalidad, la variable relativa al estrés es denominada "estrés parental adaptativo" y la relativa al conflicto se denomina "conflicto parental adaptativo", con la intención de reflejar el matiz positivo y protector del desarrollo que ambas variables pueden poseer si se gestionan adecuadamente en la dinámica familiar.

Dentro del apoyo social, también se identifica la influencia del capital social (McPherson, Kerr, Morgan, McGee, Cheater, McLean y Egan, 2013) que una familia recibe para la práctica de sus tareas de crianza y que se concreta en la disponibilidad de medidas políticas de apoyo a la función parental, tales como medidas de conciliación laboral, el apoyo económico y fiscal, la posibilidad de recurrir a programas específicos de apoyo parental, etc. Además, cabe destacar la relación familia-escuela, ya que la relación coordinada de ambos contextos, confluye en la promoción del desarrollo infantil. Asimismo, en el periodo perinatal se pueden incluir en la ecología de la parentalidad todas aquellas variables protectoras de una programación fetal negativa proveniente del contacto con neurotóxicos, estrés materno, etc. (Roncallo, Barreto y Sánchez de Miguel, 2018).

La cuarta dimensión, también calificable como dimensión transversal dado que afecta a las tres anteriores, es la estructura, definida por Pourtois y Desmet (2006) como la necesidad más básica del ser humano en el marco del paradigma de las doce 
necesidades. Un contexto familiar estructurado provee a niños y niñas un entorno predecible que genera seguridad y que, fundamentalmente, se concreta en la existencia de rutinas de interacción en todos los ámbitos de la vida familiar, desde los hábitos de alimentación hasta la presencia constante del juego u otras actividades de interacción positivas. A las rutinas se añaden los rituales asociados a ellas que incluyen un significado emocional y cultural que las enriquece y las hace más potentes e influyentes en el desarrollo infantil. La transcendencia de las rutinas y rituales como elementos estructuradores del contexto familiar queda reflejada en los trabajos de Spagnola y Fiese (2007), y de Fiese y Fisher (2019). En el contexto actual de la pandemia de covid-19, Glynn et al. (2021) han puesto de manifiesto el papel protector de un entorno familiar estructurado en rutinas sobre la salud mental de niños y niñas de cuatro años de edad.

Finalmente, partiendo del marco teórico expuesto anteriormente, se presenta a continuación el programa Descubre tu potencial para educar a tus hijas e hijos, que pretende alcanzar los siguientes objetivos:

- Evaluar la calidad de los contextos familiares de las familias participantes.

- Identificar los factores protectores y los factores de riesgo de cada una de las familias.

- Evaluar el desarrollo infantil de los niños y niñas pertenecientes a las familias participantes en el programa.

- Facilitar que las familias evaluadas construyan competencias parentales potenciadoras de los factores protectores y reductoras de los factores de riesgo.

- Prevenir que los factores de riesgo se conviertan en desventajas significativas para estas familias.

- Evaluar la construcción de nuevas competencias por parte de las familias participantes que promuevan un adecuado desarrollo psicológico de sus hijas e hijos.

\section{Método}

La propuesta de intervención contenida en este programa sigue una orientación de promoción de las competencias parentales basada en un enfoque constructivista. Desde el punto de vista metodológico se ubica en la orientación basada en la evidencia que utiliza un diseño de intervención-orientación psicoeducativa de corte longitudinal con medidas repetidas.

\subsection{Participantes}

Cualquier familia con niños y niñas entre cero y tres años usuaria de un servicio municipal puede ser susceptible de invitación, orientación o derivación al programa. En este estudio, fueron evaluadas 89 familias entre los años 2018 y 2019. Las personas profesionales tuvieron a su disposición el cartel, los dípticos informativos sobre el programa para facilitar la información necesaria a las familias que a su juicio pudieran beneficiarse del mismo. Como norma general se aplicaron los siguientes criterios de derivación y exclusión.

\section{Criterios de derivación}

- Familias que verbalizan impotencia. Por ejemplo: "No puedo con ella o con él", ansiedad ante el llanto del bebé (o-10 meses) y las rabietas (a partir de doce meses).

- Familias resistentes a los cambios cuando hay una propuesta de cambio de hábitos por parte del servicio de pediatría (hábitos de sueño, de alimentación, normas y límites, etc.), y/o por parte de las escuelas de educación infantil (normas y límites, responsabilidades, etc.), ludotecas u otros servicios.

- Familias que, debido a su elevada formación, por ejemplo, o por la lectura de recursos relacionados con la crianza y educación, se anticipan a lo que esperan del desarrollo de sus hijos e hijas.

- Familias asiduas al servicio de pediatría (más de dos veces por semana).

- Madres y padres adolescentes con hijos e hijas.

- Madres y/o padres primerizos a partir de 42 años.

- Familias poco proactivas al juego, la actividad y la interacción con sus hijos e hijas.

- Familias con escasa red de apoyo social y/o familiar.

- Familias que por su situación sociocultural se podrían beneficiar de la formación en crianza.

\section{Criterios de exclusión}

- Madres, padres y/o personas cuidadoras con problemas graves de salud mental y/o adicciones no tratadas que les genere incapacidad para poder beneficiarse del programa.

- Familias con hijos e hijas en situación de desprotección en cualquiera de sus tipologías, familias en situación de exclusión social y en las que se esté produciendo de forma activa violencia de género $u$ otras formas de abuso que requieran abordaje específico.

- Familias con bajo nivel de compresión y comunicación en lengua castellana.

\subsection{Procedimiento}

Fase 1: Difusión

- Información del programa desde el Servicio de Infancia y Familia a haurreskolak, ludotecas, senidegune, centros de salud, etc. Se utiliza 
una presentación dirigida a los profesionales encargados de derivar a las familias al servicio.

- Información a las familias sobre el contenido del programa. La llevan a cabo los profesionales que pueden derivar familias al servicio, apoyándose en un documento de difusión del programa.

\section{Fase 2: Registro en el programa de las familias participantes}

- Las familias interesadas en participar rellenan y firman el compromiso informado dando autorización para su participación y el tratamiento de sus datos de carácter personal.

- La participación de las familias es gratuita y voluntaria. La ejecución del programa se atiene al código deontológico vigente en el ámbito de la intervención social con familias y la propuesta es aprobada por el comité ético correspondiente.

\section{Fase 3: Entrevista inicial de evaluación de la calidad} del contexto familiar

- La persona profesional psicóloga establece contacto con la familia que ha autorizado la evaluación para concretar el día y la hora de la entrevista. Se estima que la duración de la visita oscila entre hora y media y una hora y tres cuartos. Se recuerda a la familia que al menos una de las personas adultas responsables del niño o niña deberá estar presente durante la entrevista.

- La persona profesional realiza igualmente la evaluación del desarrollo infantil a través de la observación directa y de la información proporcionada por madres, padres o personas responsables de la crianza.

\section{Fase 4: Entrevista de devolución a la familia del resultado de la evaluación}

- La persona profesional que atiende a cada familia realiza un informe escrito que incluirá la descripción de los factores protectores y de riesgo detectados y las orientaciones generales y específicas dirigidas a la mejora de la calidad del contexto familiar. Las orientaciones también incluyen las derivadas, cuando proceda, de la evaluación del desarrollo infantil; especialmente cuando en la exploración se detecte algún indicador específico de desarrollo atípico.

- Si se constata la estabilidad de algún indicador de desarrollo atípico, se procede a invitar a la familia a la realización de una evaluación exhaustiva del desarrollo psicológico llevada a cabo en el centro educativo o servicio social correspondiente.

- La devolución del informe se realiza de forma presencial en el domicilio familiar por parte de la persona profesional asignada a cada caso, a través de una entrevista con cita previa. La estructura y contenido del informe se realiza siguiendo los criterios expuestos en el manual del instrumento de evaluación.
- Las familias también son informadas con precisión de otros servicios municipales de los que se pudieran beneficiar.

\section{Fase 5: Entrevista de seguimiento}

- En el plazo de tres meses, la psicóloga realiza la entrevista a domicilio con la familia para conocer la evolución de la puesta en práctica de las estrategias de mejora.

- Para la evaluación del grado de instauración de las orientaciones recibidas por las familias, en la entrevista de devolución se utiliza un cuestionario específico.

- Finalmente, cada familia realiza una valoración sobre la calidad del programa.

\section{Fase 6: Oferta de asesoramiento 'online'}

- Una vez realizada la entrevista final de seguimiento, las familias disponen de un servicio online personalizado de asesoramiento sobre cuestiones de crianza. El servicio es atendido por los mismos profesionales que han llevado a cabo el programa y tiene una duración de un año a partir de la finalización de la sesión de devolución de resultados.

- En los casos en los que no se compruebe un cambio estable de rutinas relativas a las orientaciones ofrecidas a las familias en la fase de seguimiento, se vuelve a insistir a las familias en la importancia de las orientaciones y se les ofrece la opción de consultar sobre ello en el servicio de asesoramiento online.

\section{Fase 7: Evaluación del impacto del programa}

- La metodología de evaluación consiste en diseño longitudinal de intervención.

- Los datos de las evaluaciones individuales de cada familia y cada niño, llevados a cabo en la entrevista inicial (Tiempo 1) y en la entrevista de seguimiento (Tiempo 2), se procesan en una matriz de datos global con el fin de analizar si hay o no un impacto significativo del programa en el grupo de familias que han participado.

- Desde el punto de vista técnico, se aplican los análisis estadísticos que permitan obtener evidencias del impacto del proceso de evaluacióndevolución-orientación sobre la consolidación y construcción de competencias parentales promotoras del desarrollo psicológico. La evaluación del programa se completa con el registro de datos relativos a la autopercepción de las familias sobre el impacto del programa y con su valoración de satisfacción sobre el proceso.

\subsection{Instrumentos}

- Escala de Evaluación Familiar Haezi-Etxadi (Arranz et al., 2012) adecuada para niñas y niños entre 18 
y 30 meses de edad. A través de este instrumento se obtienen datos de observación directa, de una entrevista y de dos cuestionarios: uno de pareja y otro individual. El instrumento de evaluación está descrito en su manual donde se explicita el procedimiento de aplicación y procesamiento de datos.

- Inventario de Desarrollo Batelle (Newborg, Stock y Wnek, 2011): se utiliza la prueba de cribado que contiene un total de 96 ítems para evaluar las áreas personal, social, adaptativa, motora, de comunicación y cognitiva.

- Cuestionario sobre el uso de las nuevas tecnologías (pantallas), elaborado ad hoc para su uso dentro del mismo programa.

- Informe de devolución de resultados con orientaciones a las familias, elaborado ad hoc para su uso dentro del mismo programa.

- Cuestionario de seguimiento de las orientaciones elaborado ad hoc para su uso dentro del mismo programa.

- Cuestionario de valoración del programa por parte de las familias elaborado ad hoc para su uso dentro del mismo programa.

- Los datos sociodemográficos de la familia se recogen en el protocolo previo de la Escala de Evaluación Familiar Haezi-Etxadi.

\section{Resultados}

Los resultados de la primera implementación piloto del programa fueron publicados en la revista Educació Social (Arranz et al., 2016b) y traducidos y publicados posteriormente en la revista Early Child Development and Care (Arranz et al., 2019). Los resultados más significativos de la segunda fase de implementación del programa, correspondiente a los años 2018 y 2019, serán presentados en este artículo en dos bloques. En el primer bloque, previa presentación de los datos sociodemográficos, se presentan datos sobre la calidad de los contextos familiares, el desarrollo infantil, el uso de las nuevas tecnologías, las consultas realizadas por las familias en el servicio online y la valoración del programa por parte de las familias. En el segundo bloque, siguiendo el enfoque de un proceso de I+D+i, se presentarán las propuestas de mejora para futuras ediciones, que se derivan del análisis cuantitativo y cualitativo de la implementación del programa que se presenta en este artículo.

\subsection{Bloque primero}

Incluye resultados sobre los datos sociodemográficos, calidad de los contextos familiares, desarrollo infantil, uso de nuevas tecnologías, servicio de orientación online y valoración del programa por parte de las familias.

\section{Datos sociodemográficos}

La estructura de las 89 familias se corresponde con la siguiente distribución: tradicional (83; 93,3\%), monoparental ( $4 ; 4,5 \%)$, divorciada $(1 ; 1,1 \%)$ y reconstituida $(1 ; 1,1 \%)$. El nivel de estudios de los padres ( $N=85$, ya que hay cuatro familias monoparentales de madre) se corresponde con la siguiente distribución: estudios elementales ( $5 ; 5,9 \%$ ), bachiller (7; 8,2 \%), formación profesional (26, 30,6\%), diploma universitario $(12 ; 14,1 \%)$, grado universitario (32; 37,6\%) y doctorado (3; 3,6\%). El nivel de estudios de la madre corresponde a la siguiente distribución: estudios elementales $(8,9 \%)$, bachiller $(5 ; 5,6 \%)$, formación profesional (16;18\%), diploma universitario (11; $12,3 \%)$, grado universitario $(45 ; 50,6 \%)$ y doctorado ( $4 ; 4,5 \%)$. El nivel mensual de ingresos de las familias participantes corresponde a la siguiente distribución: menos de 1.000 euros ( $5 ; 6 \%)$, entre 1.000 y 2.500 euros (23; $26 \%$ ), entre 2.500 y 5.000 euros ( $43 ; 48 \%$ ), mayor de 5.000 euros (3; $3 \%$ ) y no informan (15; 17\%). La edad media de las madres fue de 34,33 años (desviación estándar $=2,55$ ) y de los padres 37,16 años (desviación estándar = 3,57).

Con relación a los hijos e hijas, puesto que en algunas familias había más de un menor entre cero y tres años, se evaluó a un total de cien niños y niñas ( $51 \%$ niñas). El rango de edad corresponde a la siguiente distribución: 0-6 meses (5,5\%), 7-12 meses (11,11\%), 13-18 meses (17,17\%), 19-24 meses $(25,25 \%), 25-30$ meses $(17,17 \%), 31-36$ meses $(15,15 \%), 37-42$ meses ( $8,8 \%), 43-48$ meses $(2,2 \%)$. La edad media en meses fue de 23,71 (desviación estándar =9,68).

\section{Datos sobre la calidad de los contextos familiares}

Los datos correspondientes a los años 2018 y 2019 se presentan en la Tabla 1 y los relativos a la totalidad de las familias evaluadas en el programa, incluyendo la prueba piloto, se muestran en la Tabla 2. 
Tabla 1. Porcentaje de familias clasificadas según el nivel de calidad del contexto familiar en las tres subescalas y sus correspondientes factores ( $N=89$ familias; años 2018 y 2019).

\begin{tabular}{|l|c|c|c|c|}
\hline \multirow{2}{*}{} & \multicolumn{3}{|c|}{ Nivel de calidad (\%) } \\
\cline { 2 - 5 } & Muy Alta & Alta & Media & Baja \\
\hline Subescala EDCL & $\mathbf{7 3 , 0}$ & $\mathbf{2 2 , 5}$ & $\mathbf{4 , 5}$ & - \\
\hline Materiales de Estimulación del Aprendizaje (MEA) & 76,4 & 23,4 & - & - \\
\hline Potencial de Juego (PJ) & 70,8 & 14,6 & 14,6 & - \\
\hline Estimulación del Desarrollo Cognitivo (EDC) & 78,7 & 9,0 & 12,3 & - \\
\hline Estimulación del Desarrollo Lingüístico (EDL) & 74,1 & 20,2 & 5,7 & \\
\hline Subescala EDSE & $\mathbf{8 7 , 6}$ & $\mathbf{1 2 , 4}$ & - & - \\
\hline Expresividad Emocional (EE) & 83,1 & 16,9 & - & - \\
\hline Establecimiento de Límites y Frustración Óptima (ELFO) & 75,3 & 19,1 & 5,6 & - \\
\hline Potenciación de la Autoestima y la Autonomía (PAA) & 43,9 & 38,2 & 15,7 & 2,2 \\
\hline Observación de la Interacción Con el Niño/a (OIN) & 100,0 & - & - & - \\
\hline Subescala OEFCS & $\mathbf{8 7 , 6}$ & $\mathbf{1 1 , 3}$ & - & - \\
\hline Calidad del Entorno Físico (CEF) & 100,0 & - & - & - \\
\hline Implicación del Padre o segunda figura de crianza (IP) (*4,5\% no aplicable) & 76,4 & 13,5 & 5,6 & - \\
\hline Calidad del Cuidado Sustituto (CCS) (*73,1\% no aplicable) & 26,9 & - & - & - \\
\hline Relaciones con la Familia Extensa y red social de amistades y servicios (RFE) & 87,6 & 9,0 & 3,3 & - \\
\hline Estabilidad de las Relaciones Sociales Infantiles e interés parental en las mismas (ERSI) & 73,0 & 22,5 & 3,4 & 0,1 \\
\hline Relaciones con la Escuela (RE) (*26,9\% no aplicable) & 69,7 & 2,3 & - & 1,1 \\
\hline Diversidad de Experiencias (DE) & 92,1 & 4,5 & 3,4 & - \\
\hline Exposición al Conflicto Familiar (ECF) (*1,1\% no aplicable) & 83,2 & 13,5 & - & 2,2 \\
\hline Estrés Parental (EP) & 64,0 & 20,3 & 13,5 & 2,2 \\
\hline
\end{tabular}

Nota: $\mathrm{EDCL}=$ Estimulación del Desarrollo Cognitivo y Lingüístico; EDSE = Estimulación del Desarrollo Socio-Emocional; OEFCS = Organización del Entorno Físico y del Contexto Social.

Tabla 2. Porcentaje de familias clasificadas según el nivel de calidad del contexto familiar en las tres subescalas y sus correspondientes factores $(N=139$ familias; años 2016, 2018 y 2019)

\begin{tabular}{|l|c|c|c|c|}
\hline \multirow{2}{*}{} & \multicolumn{3}{|c|}{ Nivel de calidad (\%) } \\
\cline { 2 - 5 } & Muy Alta & Alta & Media & Baja \\
\hline Subescala EDCL & $\mathbf{7 3 , 5}$ & $\mathbf{2 3 , 2}$ & $\mathbf{3 , 2}$ & - \\
\hline Materiales de Estimulación del Aprendizaje (MEA) & 86,2 & 12,7 & - & - \\
\hline Potencial de Juego (PJ) & 61,4 & 20,3 & 14,3 & 4,0 \\
\hline Estimulación del Desarrollo Cognitivo (EDC) & 82,3 & 8,5 & 9,1 & - \\
\hline Estimulación del Desarrollo Lingüístico (EDL) & 77,0 & 18,1 & 2,9 & 2,0 \\
\hline Subescala EDSE & $\mathbf{8 4 , 8}$ & $\mathbf{1 4 , 2}$ & $\mathbf{1 , 0}$ & - \\
\hline Expresividad Emocional (EE) & 86,5 & 11,4 & 2,0 & - \\
\hline Establecimiento de Límites y Frustración Óptima (ELFO) & 76,6 & 17,5 & 3,8 & 2,0 \\
\hline Potenciación de la Autoestima y la Autonomía (PAA) & 45,9 & 36,1 & 11,8 & 6,1 \\
\hline Observación de la Interacción con el Niño/a (OIN) & 100,0 & - & - & - \\
\hline Subescala OEFCS & $\mathbf{8 7 , 6}$ & $\mathbf{1 1 , 3}$ & - & - \\
\hline Calidad del Entorno Físico (CEF) & 100,0 & - & - & - \\
\hline Implicación del Padre o segunda figura de crianza (IP) (*4,5\% no aplicable) & 80,2 & 11,7 & 5,8 & - \\
\hline Calidad del Cuidado Sustituto (CCS) (*37,6\% no aplicable) & 63,4 & - & - & - \\
\hline Relaciones con la Familia Extensa y red social de amistades y servicios (RFE) & 90,8 & 6,5 & 2,6 & - \\
\hline Estabilidad de las Relaciones Sociales Infantiles e interés parental en las mismas (ERSI) & 65,5 & 31,2 & 1,7 & 1,0 \\
\hline Relaciones con la Escuela (RE) (*13,6\% no aplicable) & 84,8 & 1,1 & - & 0,5 \\
\hline Diversidad de Experiencias (DE) & 94,0 & 4,2 & 1,7 & \\
\hline Exposición al Conflicto Familiar (ECF) (*0,6\% no aplicable) & 79,6 & $\mathbf{1 2 , 7}$ & 3,0 & 4,1 \\
\hline Estrés Parental (EP) & 64,0 & 19,1 & 4,7 & 2,1 \\
\hline
\end{tabular}

Nota: $E D C L=$ Estimulación del Desarrollo Cognitivo y Lingüístico; EDSE= Estimulación del Desarrollo Socio-Emocional; OEFCS= Organización del Entorno Físico y del Contexto Social. 
Los niveles de desarrollo infantil, de la evaluación realizada a los cien niños y niñas participantes ( $51 \%$ niñas), corresponden a la siguiente distribución: Desarrollo cognitivo (Alto, 47,47\%; Medio, 45,45\%; Bajo, 4,4\%; No aplicable 4,4\%), Desarrollo Comunicativo (Alto, 37,37\%; Medio, 47,47\%; Bajo, 12,12 \%; No aplicable, 4,4\%), Desarrollo Motor (Alto, 33,33\%; Medio, 49,49\%; Bajo, 14,14\%; No aplicable, 4,4\%), Desarrollo Personal-Social (Alto, 29,29\%; Medio, 43,43\%; Bajo, 24,24\%; No aplicable, 4,4\%), Desarrollo Adaptativo (Alto, 32,32\%; Medio, 45,45\%; Bajo, 19,19\%; No aplicable, 4,4\%).

\section{Datos sobre el contacto de los niños y niñas con pantallas}

En lo que se refiere a la frecuencia de exposición, los datos se corresponden con la siguiente distribución: el $63 \%$ de las familias no ofrecen o exponen a sus hijos e hijas a las pantallas, mientras que el $37 \%$ ofrecen o exponen a sus hijos e hijas a las pantallas más de una vez al día. En cuanto al objetivo del uso de las pantallas, los datos se corresponden con la siguiente distribución: el $\mathbf{2 4 , 7} \%$ de las familias las usan para gestionar situaciones cotidianas que les suponen un conflicto (negativa a comer, a dormirse, rabietas y otras) y el 75,3\% las utiliza de forma lúdica.

En lo que se refiere a la cantidad de tiempo de exposición, los datos se corresponden con la siguiente distribución: el 59,5\% de las familias no dejan que sus hijos e hijas estén más de media hora expuestos a las pantallas, y cuando las utilizan lo hacen acompañados/as, el 40,5\% de las familias dejan que sus hijos e hijas estén más de media hora expuestos a las pantallas y cuando las utilizan lo hacen solos/as. En cuanto a la valoración que madres y padres hacen sobre el riesgo de la exposición a pantallas en el desarrollo infantil, los datos se corresponden con la siguiente distribución: el $2,2 \%$ cree que es beneficioso, el $79,7 \%$ cree que no beneficia y el $18,1 \%$ se sitúa en un punto medio. Por otra parte, el $44,9 \%$ cree que perjudica, el 39,3\% cree que no perjudica y el $15,8 \%$ se sitúa en un punto medio.

\section{Datos sobre las consultas de las familias en el servicio online}

El $64 \%$ de las familias (57 en total) han consultado sobre uno o varios temas en relación con la crianza y educación de sus hijas e hijos; los datos se corresponden con la siguiente distribución: 13 consultas sobre gestión de rabietas, 10 sobre establecimiento de normas y límites, 11 sobre gestión de los celos, 6 sobre autorregulación emocional, 5 sobre hábitos de sueño, 5 sobre control de esfínteres, 4 sobre conflictos entre hermanos y hermanas, 3 sobre hábitos de alimentación, 3 sobre fomento de la autonomía, 3 sobre la socialización en la infancia, 3 sobre juego en familia, 2 sobre andamiaje del lenguaje, 2 sobre bibliografía de guías de crianza, 2 sobre incorporación al centro escolar, 2 sobre otros temas, 1 sobre criterios de afrontamiento de la separación de la pareja, miedos en la infancia, mordiscos, fomento de la autoestima, comunicación asertiva, uso de pantallas, gestión de la competitividad, apego, corresponsabilidad, destete, organización familiar, metodología Emmi Pikler, e información sobre servicios municipales para familias monoparentales.

Datos sobre la valoración del programa por parte de las familias

De las 89 familias participantes en el programa, $76(84,4 \%)$ han contestado al cuestionario sobre la valoración del mismo. En lo que se refiere a la valoración relativa a la calidad del programa, las familias han mostrado una puntuación media de 26,7 puntos sobre un máximo posible de 28 puntos. En cuanto a la valoración de la eficacia del programa, las familias han mostrado una puntuación media de 10 sobre un máximo posible de 12 puntos. En cuanto a la satisfacción sobre su participación en el programa, las familias han mostrado una puntuación media de 11,5 puntos, sobre una puntuación máxima de 12 puntos. La media de valoración general del programa alcanza una puntuación de 46,6 puntos, sobre un máximo posible de 52 puntos.

Las familias han realizado diversas sugerencias sobre la aplicación del programa; ocho familias sugieren aumentar el número de visitas domiciliarias, siete proponen aumentar el rango de edad hasta los seis años, tres de ellas plantean la conveniencia de realizar encuentros entre las familias participantes, dos familias señalan la necesidad de más ejemplos prácticos, una familia sugiere que el primer contacto se haga con mayor antelación, una familia sugiere que se haga más y mejor difusión del programa, una familia sugiere que el seguimiento se haga cada seis meses en vez de cada tres, una familia sugiere que el seguimiento se haga cada doce meses, una familia sugiere la realización de programas centrados en la pareja, una familia sugiere la realización de un informe más concreto y personal, una familia sugiere ampliar la disponibilidad horaria para la realización de la entrevistas, una familia sugiere incluir en la última sesión una valoración del niño/a $y$, finalmente, una familia sugiere practicar las pautas con la presencia de la psicóloga.

\subsection{Bloque segundo}

Este bloque incluye resultados derivados de la implementación del programa y que se han incorporado al mismo llevando a cabo un proceso de Investigación + Desarrollo + Innovación. En función de los resultados obtenidos en la aplicación del programa, de la experiencia de los profesionales que han visitado 


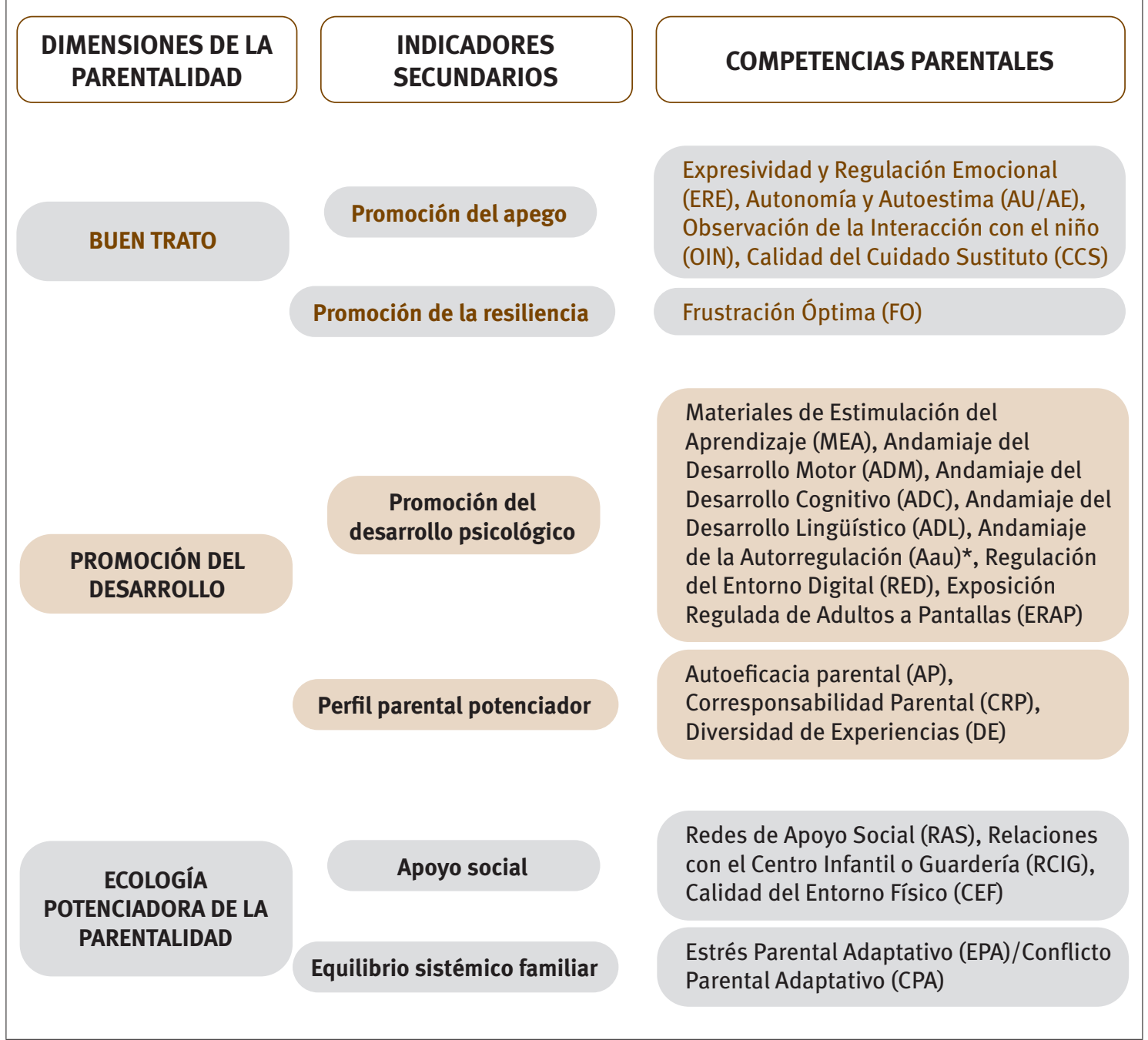

* Para versiones 18-35 meses y 36-59 meses.

Fuente: Elaboración propia.

a las familias en sus hogares y de la valoración técnico-científica llevada a cabo por la comisión de seguimiento del programa, formada por el Servicio de Infancia y Familia del Ayuntamiento de Vitoria-Gasteiz y el Grupo Haezi-Etxadi de la UPV/EHU, se toma una serie de decisiones, que serán adecuadamente justificadas en la sección de discusión de este trabajo.

Decisiones relativas a los instrumentos utilizados en el programa

- Sustituir la Escala de Evaluación Familiar HaeziEtxadi a dos años por cuatro nuevas escalas que se derivan de ella: HEFAS (Haezi Etxadi Family Assessment Scale) 0-9 meses, 10-17 meses, 18-35 meses y 36-59 meses. La estructura de competencias evaluada a través de las nuevas escalas se refleja en el Gráfico 1.

- Incluir el Instrumento CDP+ (Cuestionario de Dimensiones de la Parentalidad Positiva)
(Arranz-Freijo y Barreto-Zarza, 2020) que explora creencias y actitudes sobre la estructura de competencias evaluadas con las escalas HEFAS.

- Sustituir el Inventario de Desarrollo Batelle (Newborg, Stock y Wnek, 2011) por el ASQ-3, versión en español (Squires, Bricker y Twombly, 2009).

- Incluir un nuevo instrumento sobre el impacto de la pandemia de la covid-19 en la vida familiar.

- Incluir un nuevo modelo de informe de devolución, que contiene orientaciones para el establecimiento de rutinas interactivas positivas derivadas de los ítems de las escalas HEFAS en los que las familias obtienen una puntuación negativa.

- Incluir una nueva ficha de seguimiento de las orientaciones específicas ofrecidas a cada familia en la sesión de devolución. 
Decisiones relativas al diseño e implementación del programa

- Modificar el diseño del programa al denominado diseño longitudinal con medidas repetidas. Con ese fin, la psicóloga realiza un seguimiento telefónico a los dos y cuatro meses desde la entrevista de devolución de resultados de la primera evaluación y de la entrega de orientaciones para incorporar nuevas rutinas, y a los seis meses, en la fase de evaluación del programa (Tiempo 2), se vuelven a aplicar los mismos instrumentos que se aplicaron en la evaluación inicial (Tiempo 1).

- Incluir como actividad final de la entrevista de devolución el visionado conjunto y la puesta en común, del vídeo Pegados o despegados, relativo al uso de dispositivos electrónicos en la infancia temprana, realizado por el Servicio de Infancia del Ayuntamiento de Vitoria-Gasteiz (Arranz-Freijo et al., en prensa).

- Adaptar la propuesta del programa a los criterios que aparecen en la Guía de buenas prácticas para el diseño e implementación de programas de parentalidad positiva, elaborado por Rodrigo et al. (2015) y el grupo de expertos en el campo, que trabajan en el diseño de políticas familiares de parentalidad positiva en el marco del convenio de colaboración entre el Ministerio de Sanidad, Consumo y Bienestar Social del Gobierno de España y la Federación Española de Municipios y Provincias. Los criterios mencionados están expuestos en el documento citado y en el protocolo interactivo al que los profesionales pueden acceder dándose de alta en el sitio web 〈www.familiasenpositivo.org〉.

- Las buenas prácticas (BP) relativas al diseño e implementación de programas se exponen a continuación:

- BP 21: fundamentar el programa en una base científica y formular objetivos claros y susceptibles de ser medidos.

- BP 22: desarrollar el programa con una metodología bien diseñada para la atención individual, grupal o comunitaria.

- BP 23: Coordinar y dinamizar el grupo de forma eficaz fomentando un clima de relación positivo en el programa grupal.

- BP 24: Evaluar el programa con criterios científicos.

- BP 25: Incorporar el programa a la comunidad y contribuir a su desarrollo. Las buenas prácticas mencionadas se ajustan igualmente a los criterios expuestos en la propuesta de Arranz et al. (2016a) elaborada para la Dirección de Política Familiar y Diversidad de la Consejería de Asuntos Sociales del Gobierno Vasco y a los criterios expuestos en el Manual para profesionales en la implementación de programas de parentalidad positiva de Asmussen (2012).
- Elaborar un manual actualizado del programa en el que se describan sus fundamentos teóricos, el procedimiento de implementación, y la definición de instrumentos, así como su aplicación, procedimiento de corrección, interpretación y procesamiento.

\section{Discusión}

A continuación, se valorarán los resultados correspondientes a las variables exploradas en las familias durante la implementación del programa (bloque primero), seguidamente se valorarán los resultados derivados de la implementación del programa y del proceso I+D+I (bloque segundo). Finalmente se expondrán las implicaciones prácticas y de acción política que se derivan de los resultados del programa.

En lo que se refiere a las variables del contexto familiar evaluadas en la segunda fase de implementación del programa -años 2018 y 2019 (bloque primero)-, procede resaltar que los datos obtenidos son fundamentalmente descriptivos, no son en un principio generalizables a la población general y poseen un valor fundamentalmente cualitativo sobre las características de la población estudiada.

\subsection{Datos sociodemográficos}

Los datos sociodemográficos muestran un perfil favorable para la práctica de la parentalidad positiva; el nivel educativo medio-alto y alto de madres y padres, que muestra un $32 \%$ de padres y un $45 \%$ de madres con estudios universitarios, constituye un predictor de una interacción familiar de calidad, sobre todo para la adecuada promoción del desarrollo cognitivo y lingüístico. La literatura identifica esta influencia preferentemente en relación con las madres; el estudio de Schady (2011) confirma este hallazgo en un estudio longitudinal con una muestra de cohorte de 2.113 niños y niñas entre 36 y 71 meses. El perfil favorable expuesto queda completado por un nivel socioeconómico medioalto y alto, con un $48 \%$ de las familias declarando unos ingresos mensuales entre 2.500 y 5.000 euros. La influencia positiva del estatus socioeconómico sobre la calidad de la crianza constituye un hallazgo sólidamente establecido en la literatura (Conger, Martin y Masarik, 2021; Roubinov y Boyce, 2017), aunque las vías de esta influencia están siendo adecuadamente estudiadas con el objetivo primordial de optimizar la crianza en familias con bajo estatus socioeconómico.

\section{Calidad de los contextos familiares}

Tomando como referencia los datos porcentuales globales de todas las familias que han participado en el programa (139 desde su inicio) se puede deducir 
que la calidad general del contexto familiar en todos los factores explorados se sitúa en niveles muy satisfactorios, siendo estos datos en un principio compatibles con el perfil sociodemográfico expuesto anteriormente. No obstante, cabe destacar la presencia de familias en niveles medios y bajos de calidad interactiva familiar, en factores como el juego (25 familias), estimulación del desarrollo cognitivo (12 familias), estimulación del desarrollo lingüístico (7 familias), establecimiento de límites y frustración óptima (8 familias), potenciación de la autoestima y la autonomía (24 familias), implicación del padre o segunda figura de crianza (8 familias), relaciones con la familia extensa y red social de amistades y servicios (12 familias), estabilidad de las relaciones sociales infantiles e interés parental en las mismas $(3$ familias), exposición al conflicto familiar ( 9 familias) y estrés parental (23 familias).

En la valoración de estos datos, parece oportuno destacar que el instrumento utilizado posee un potencial de detección de condiciones interactivas mejorables en las familias evaluadas. La consecuencia más significativa de este hecho reside en que estas familias se han beneficiado de una atención individualizada, consistente en la recepción de orientaciones específicas dirigidas a su mejora competencial para mejorar sus condiciones interactivas, con el objetivo último de aumentar el bienestar familiar y promover el desarrollo psicológico saludable de sus hijos e hijas. También procede resaltar que, dado el perfil sociodemográfico protector de las familias evaluadas, si se hubiera dado el caso de haber sido evaluadas específicamente familias con un perfil no tan favorable, probablemente los resultados de calidad interactiva familiar hubieran sido menos satisfactorios, debido al previsible aumento del estrés familiar. El aumento del estrés sostenido en el tiempo se denomina estrés tóxico (Shonkoff et al., 2012), y es una respuesta natural a una situación de mayor inestabilidad socioeconómica que es muy poco compatible con una interacción familiar promotora del desarrollo psicológico infantil.

\section{Evaluación del desarrollo infantil}

Los datos relativos a la evaluación del desarrollo infantil muestran unas distribuciones entre el $40 \%$ y el $50 \%$ de sujetos ubicados en los niveles medios, entre el $29 \%$ y el $47 \%$ de niños y niñas se ubicó en los niveles altos y, finalmente, entre el $4 \%$ y el $24 \%$ se ubicó en los niveles bajos. Con relación a las áreas evaluadas, aquella en la que se obtuvo un mayor porcentaje de niños y niñas con nivel alto fue el desarrollo cognitivo ( $47 \%$ ). Por otra parte, el área de desarrollo en la que se ubicó un mayor porcentaje de niños y niñas con nivel más bajo fue el desarrollo personal-social (24\%). Cabe destacar que aquellas familias con niños y niñas ubicados en niveles bajos se han beneficiado de una atención individualizada, que se concreta en las orientaciones específicas para promover el desarrollo de sus hijas e hijos en las diversas áreas. En aquellos casos en los que se han detectado indicadores severos de desarrollo atípico, la familia ha sido derivada para recibir una evaluación más exhaustiva del desarrollo psicológico del niño o niña. Este hecho muestra que el programa incluye entre sus potencialidades, una función de detección precoz y derivación de casos de desarrollo atípico.

\section{Contacto de los niños y niñas con pantallas}

Si se tiene en cuenta que el $37 \%$ de los niños y niñas están expuestos/as a pantallas más de una vez al día y que el $60 \%$ de los niños y niñas de la muestra se encuentran en el rango de edad entre los o y los 24 meses, se deduce que en un grupo numeroso de familias se está incumpliendo la recomendación de la OMS que aconseja la ausencia total de contacto con pantallas en esa franja de edad. La evidencia empírica sobre la influencia del uso de pantallas en el desarrollo psicológico se va enriqueciendo y matizando; el estudio de Stiglic y Viner (2019) que realiza un revisión sistemática de estudios previos, muestra una asociación moderadamente intensa entre el tiempo de usos de pantallas y niveles altos de obesidad y de síntomas depresivos; sin embargo no se encuentra evidencia entre el uso de pantallas y los trastornos alimentarios o la existencia de ideas suicidas. Por su parte, Wong et al. (2021) identifican el uso de pantallas y el bajo nivel de actividades en el exterior como factores asociados al establecimiento y progresión de la miopía en niños y niñas; señalan, además, que estos efectos han podido ser potenciados por la reciente experiencia del confinamiento vivida por las familias durante la pandemia de la covid-19.

Los datos relativos al objetivo con el que son usadas las pantallas indican que estas forman parte de las estrategias parentales para gestionar situaciones cotidianas difíciles. Aunque es comprensible su uso como distractor ocasional, no parece recomendable desde el punto de vista educativo ligar los procesos de autorregulación infantil a un objeto externo, siendo esto especialmente importante en el tramo de edad entre los o y los 2 años. Igualmente, muchas familias utilizan las pantallas con un objetivo lúdico; este hecho es más preocupante cuando los niños y niñas las utilizan solos, lo que ocurre en un $40 \%$ de los casos en la muestra estudiada. Abundando en la transcendencia evolutiva de la construcción de las competencias de autorregulación, estas se construyen con solidez cuando están apoyadas sobre una interacción social directa entre los y las bebés y sus personas cuidadoras. La hipótesis del desplazamiento por parte de los dispositivos de la interacción social directa es un tema muy relevante educativamente que está siendo investigado en la actualidad (Wan et al., 2021). Finalmente, cabe destacar que, a pesar de las prácticas parentales de cierto riesgo en relación con el uso de pantallas expuestas anteriormente, el $45 \%$ de las madres y 
padres declaran que su uso perjudica el desarrollo psicológico infantil y el $80 \%$ declara que no lo beneficia.

\section{Consultas de las familias en el servicio online}

Un grupo numeroso de familias ha hecho uso del servicio de orientación online sobre crianza, ofrecido en el marco del programa; este hecho se debe probablemente a que han desarrollado una relación de confianza con la psicóloga que les ha visitado en sus hogares. La experiencia previa del equipo Haezi-Etxadi en la implementación de programas de asesoramiento online indica que las familias recurren a él cuando conocen o tienen algún tipo de interacción previa con las personas profesionales que ofrecen el servicio (Arranz Freijo, Olabarrieta Artetxe y Martín Ayala, 2010). Esa interacción se consigue en el marco de la visita domiciliaria o cuando se programan charlas o actividades de grupo sobre parentalidad positiva en la comunidad.

La casuística sobre la que han consultado las familias durante este periodo del servicio es muy amplia y abarca 25 temas diferentes, siendo más del $50 \%$ sobre la gestión de las rabietas, acontecimiento muy frecuente en el periodo evolutivo entre los 1 y los 3 años. Como ya se resaltó en la valoración de los resultados relativos al uso de pantallas, la construcción precoz de las competencias de autorregulación es un logro evolutivo-educativo muy importante, en la medida en la que el afrontamiento parental de las rabietas aporta a niñas y niños sus primeras experiencias de autorregulación/ autocontrol emocional, un aprendizaje esencial para la adaptación exitosa al entorno que les rodea. Cabe insistir en que las familias participantes en el programa se han beneficiado de una orientación individualizada, especializada y confidencial sobre varios temas que les generaban preocupación en relación a la crianza de sus hijos e hijas.

La oferta online de intervenciones de parentalidad positiva se ha ido acrecentando en los últimos años y los resultados indican que puede constituir un apoyo muy disponible y eficaz a las tareas de crianza infantil. El metaanálisis de Nieuwboer, Fukkink y Hermanns (2013) ya evidenciaba que los programas de parentalidad a través de la web ofrecían apoyo social a los participantes y también la posibilidad de consultar con profesionales y de entrenarse en la adquisición de competencias parentales. Trabajos más recientes, como el de Boekhorst et al. (2021), muestran la eficacia de un entrenamiento en crianza para madres con altos niveles de estrés que participaron en un programa de ocho semanas de duración.

\section{Valoración del programa por parte de las familias}

Los datos indican con rotundidad que las familias han valorado muy satisfactoriamente su participación en el programa; este hecho es especialmente relevante en lo que se refiere a la valoración de su eficacia, lo que supone que las familias identifican la implementación de nuevas rutinas interactivas positivas en su crianza cotidiana y un impacto positivo en el desarrollo psicológico de sus hijas e hijos. Sin embargo, como se comentará más adelante, la evaluación rigurosa del programa requiere un cambio en la metodología practicada en esta fase del programa. Finalmente, es de destacar que las familias realizan una serie de sugerencias que se dirigen fundamentalmente a mejorar cuestiones logísticas relativas a la implementación, como el sistema de citas y, sobre todo, dirigidas a lograr una mayor implantación del programa en la población de familias ubicadas en la franja de edad entre los o y los 6 años.

En lo que se refiere a la valoración de los resultados derivados de la implementación del programa y del proceso I+D+I (bloque 2), conviene resaltar que proceden de un proceso de reflexión conjunta entre todas las personas implicadas en el programa, incluyendo familias, profesionales, políticas e investigadoras.

\subsection{Instrumentos utilizados en el programa: propuesta de mejora}

La decisión de adaptar cuatro nuevos instrumentos, HEFAS, para evaluar la calidad de los contextos familiares, se tomó para lograr un ajuste máximo entre los ítems de promoción parental del desarrollo y los logros evolutivos específicos normativos de la edad infantil en meses. El objetivo de este ajuste es lograr una evaluación más fina de las competencias parentales de andamiaje, motor, cognitivo y lingüístico y evitar que el uso de criterios de evaluación más genéricos arroje como resultado un alto porcentaje de familias en niveles muy altos de calidad del contexto familiar, tal y como ha ocurrido en los años de implementación del programa.

El objetivo expuesto afecta también al instrumento de evaluación del desarrollo utilizado hasta ahora. La inclusión del nuevo instrumento (ASQ-3) aporta una herramienta con mejores indicadores psicométricos que el anterior y permite lograr una sincronía muy alta entre los ítems de evaluación de las competencias parentales de promoción del desarrollo y los ítems de evaluación del propio desarrollo infantil. Igualmente, se evita que el uso de un instrumento centrado entre los 18 y los 30 meses de edad, penalice las puntuaciones de los niños y niñas entre los o y los 18 meses y facilite la valoración positiva de los que se encuentran en la franja entre los 30 y los 47 meses. La consecuencia final de las modificaciones realizadas redunda en favor de una clara mejora estructural y cualitativa del informe que se ofrece a las familias en la fase de devolución del programa.

Además de las competencias relativas a la promoción parental del desarrollo infantil, se han incluido o reformulado nuevas competencias que 
no se encontraban suficientemente desarrolladas en el instrumento anterior. Entre ellas, es especialmente destacable la inclusión del factor Andamiaje de la Autorregulación (AAU), presente implícitamente en todas las escalas y realizada de manera explícita en las escalas de 18-35 meses y 36-59 meses. Su inclusión era obligada dada la transcendencia evolutiva de la maduración de las funciones ejecutivas en este periodo evolutivo y la preocupación que muestran las familias sobre su capacidad para gestionar las rabietas infantiles. Otra inclusión inevitable ha sido la del factor Regulación del Entorno Digital (RED), evaluada anteriormente con un instrumento aparte, pero que debe ser ya incluida dada su presencia y estabilidad en los contextos familiares actuales. El complemento adecuado a esta competencia viene constituido por la inclusión del factor Exposición Regulada de Adultos a Pantallas (ERAP), cuyo objetivo es evaluar el modelaje parental ofrecido a niñas y niños en el uso de los dispositivos electrónicos.

Otros factores del contexto familiar incluyen la medición de la Autoeficacia Parental (AP), que refleja resultados recientes de investigación que han identificado el significativo impacto de esta variable, centrada en la autovaloración que madres, padres y personas cuidadoras realizan sobre sus competencias parentales y sobre el desarrollo infantil. Su peso aumenta cuando se constata que su promoción se ha convertido en uno de los pilares de la intervención familiar en el ámbito de la parentalidad positiva. La redefinición del antiguo factor Implicación del Padre como Corresponsabilidad Parental (CRP) aporta un enfoque de coeducación y no estigmatizador que, probablemente, facilitará una mayor implicación de los padres en el proceso de crianza infantil. Finalmente, es importante resaltar que las denominaciones de Estrés Parental Adaptativo (EPA) y Conflicto Parental Adaptativo (CPA) resultan técnicamente más precisas y mucho más compatibles con el enfoque no centrado en el déficit de la perspectiva teórica de la parentalidad positiva.

Cabe destacar, finalmente, que una consecuencia significativa del diseño de cuatro nuevos instrumentos de evaluación del contexto familiar reside en el hecho de poner a disposición de las personas profesionales que trabajen en el ámbito de la intervención familiar unas nuevas herramientas para potenciar sus recursos de evaluación, que puedan servir para diseñar intervenciones basadas en una buena detección de las problemáticas y necesidades de las familias.

\subsection{Diseño e implementación del programa: propuesta de mejora}

La modificación más significativa en lo que se refiere al diseño del programa es de mucha relevancia porque se refiere a la evaluación de su impacto con un enfoque longitudinal de medidas repetidas. La nueva propuesta del diseño del programa quedaría reflejada de la siguiente manera: en Tiempo 1 se realiza la primera evaluación del contexto familiar y el desarrollo infantil. Posteriormente, se hace la entrevista de devolución de resultados en la que se entregan las orientaciones para incorporar las nuevas rutinas en función de los resultados obtenidos. A los dos y cuatro meses desde la entrevista de devolución, la psicóloga realiza un seguimiento telefónico sobre posibles dudas y sobre la incorporación de las nuevas rutinas. Finalmente, a los seis meses, en Tiempo 2, se evalúa nuevamente el contexto familiar y el desarrollo infantil para conocer si verdaderamente se instauraron las nuevas rutinas.

Esta mejora cualitativa se debe al desarrollo de los cuatro nuevos instrumentos de calidad del contexto familiar (HEFAS) que van a permitir evaluar el mismo con mayor precisión en Tiempo 2. También la evaluación del desarrollo infantil en Tiempo 2 mejora ostensiblemente con el uso del ASQ-3. El nuevo diseño contiene, por otra parte, una novedad metodológica importante al incluir tres medidas de validez convergente a las llevadas a cabo por las escalas HEFAS; una de ellas es el CDP+, centrado específicamente en la evaluación de todas las competencias incluidas en la nueva propuesta. Otra medida es la ficha de seguimiento que se provee a las familias en el informe de devolución y en la que las familias registrarán la incorporación de nuevas rutinas interactivas relativas a los ítems en los que obtuvieron en Tiempo 1 una valoración no favorable. La tercera medida convergente es la autopercepción que las familias realizan sobre el impacto del programa en la encuesta de satisfacción que cumplimentan al concluir.

Finalmente, cabe señalar como limitación de la versión renovada del programa el hecho de que los nuevos instrumentos diseñados para la evaluación del contexto familiar, escalas HEFAS, deben ser analizados desde la perspectiva de sus propiedades psicométricas, aunque estén apoyados en evidencias científicas en el ámbito de la parentalidad positiva y aunque procedan de un instrumento matriz que ya estaba validado (Velasco et al., 2014).

Como conclusión de la discusión realizada, se exponen en forma de recomendaciones las implicaciones prácticas y de acción política derivada de los datos obtenidos en el programa Descubre tu Potencial para Educar a tus Hijos e Hijas y derivadas igualmente, de su proceso de implementación desde la perspectiva de Investigación + Desarrollo + Innovación.

\subsection{Recomendaciones}

A partir de todo lo expuesto, es posible establecer una serie de recomendaciones:

- Utilizar el enfoque teórico-metodológico de las dimensiones de la parentalidad como marco 
de referencia común de la acción política en el ámbito de la parentalidad positiva.

- Extender el rango de variabilidad relativa al nivel socioeconómico y educativo de las familias participantes.

- Potenciar la derivación de familias al programa desde los ámbitos sanitario, judicial, social y educativo.

- Potenciar la incorporación del programa a la comunidad y difundir su disponibilidad para las familias residentes en el Ayuntamiento de Vitoria-Gasteiz.

- Llevar a cabo acciones de difusión y de formación dirigidas a las familias para la promoción de las competencias parentales asociadas a los factores evaluados en el programa y que generan las siguientes áreas de atención prioritaria (niveles 1, 2 у 3$):$

- Estrés parental adaptativo (1).

- Andamiaje del desarrollo cognitivo (juego) (1).

- Autorregulación emocional, cognitiva y comportamental (1).

- Autoeficacia parental (1).

- Regulación del entorno digital (1).

- Exposición regulada de adultos a pantallas (1).
- Autoestima autonomía (1).

- Conflicto parental adaptativo (2).

- Frustración óptima (2).

- Corresponsabilidad parental (2).

- Conocimiento de los hitos del desarrollo psicológico infantil (3).

- Andamiaje del desarrollo lingüístico (3).

- Redes de apoyo social (3).

- Relaciones con el centro infantil o guardería (3).

- Poner a disposición de la comunidad profesional los nuevos instrumentos de evaluación de la calidad del contexto familiar (escalas HEFAS).

- Formar a las personas profesionales que trabajan en el ámbito de intervención familiar en el uso de instrumentos de evaluación del contexto familiar.

- Formar a las personas profesionales que trabajan en el ámbito de la intervención familiar en el uso de instrumentos de evaluación del desarrollo psicológico infantil.

- Poner a disposición de las familias participantes en los programas presenciales municipales de parentalidad positiva y de apoyo familiar, un servicio de apoyo online con el objetivo de dar apoyo individualizado en un contexto de confianza y confidencialidad. 


\section{Referencias bibliográficas}

ANAUT, M. y CYRULNIK, B. (2014): Résilience. De la recherche à la pratique, París, Odile Jacob.

ARRANZ-FREIJO, E. (2002): "Conflicto: antropología, psicología y educación”, XV Congreso de Estudios Vascos: Ciencia y cultura vasca y redes telemáticas, Donostia, Eusko lkaskuntza, pp. 241-252.

ARRANZ-FREIJO, E. y BARRETO-ZARZA, F. (2020): Cuestionario de dimensiones de la parentalidad, Universidad del País Vasco (UPV/EHU).

- (2021): Programa Criando en Equipo. Intervención psicoeducativa para familias en situación de vulnerabilidad con niños y niñas entre o y 6 años, Cruz Roja Española.

ARRANZ-FREIJO, E.; BARRETO-ZARZA, F.B.; ETXANIZ ARANZETA, A.; OLABARRIETA ARTETXE, F.; RONCALLO ANDRADE, C.P.; ACHA, J. y GARCIA, M.D. (en prensa): "Bebés y pantallas digitales: una propuesta educativa audiovisual desde la parentalidad positiva", Educació Social. Revista d'Intervenció Socioeducativa.

ARRANZ-FREIJO, E.; OLABARRIETA, F.; MANZANO, A.; BARRETO, F.; RONCALLO, C.; SÁNCHEZMURCIANO, M.; REKAGORRI, J. y GARCÍA M.D. (2016b): "Evaluación y educación preventiva de familias desde las claves de la parentalidad positiva", Educació Social. Revista d'Intervenció Socioeducativa, 64, pp. 11-28.
EIJO, E.; OLABARRIETA, F.; MANZANO, A.; BARRETO, F.B.; RONCALLO, C.P.; SÁNCHEZ MURCIANO, M.; REKAGORRI, J. y GARCIA, M.D. (2019): “Assessment and preventive education for families, based on the principles of positive parenting", Early Child Development and Care, 189(5), pp. 792-801.

ARRANZ-FREIJO, E.; OLABARRIETA ARTETXE, F.; MANZANO FERNANDEZ, A.; MARTIN AYALA, J.L. y GALENDE
PEREZ, N. (2012): “The Etxadi Gangoiti Scale: a Proposal for Evaluating the Family Contexts of Two-year-old Children", Revista de Educación, 358, pp. 218-237.

ARRANZ-FREIJO, E.B.; OLABARRIETA, F.; MANZANO, A.; MARTÍN, J.L.; CRUZ, N. y ETXANIZ, A. (2016a): "Assessment of positive parenting programmes in the autonomous region of the Basque Country (Spain)", Psychosocial Intervention, 25(2), pp. 127-134.

ARRANZ FREIJO, E; OLABARRIETA ARTETXE, F. y MARTÍN AYALA, J.L. (2010): "Educating from the family: A Proposal to connect homes and institutions", en Unceta, A. y Medrano, C. (eds.), Equality, Equity, and Diversity, Reno, Center for Basque Studies, pp.159-174.

ARRANZ-FREIJO, E. y RODRIGO LÓPEZ, M.J. (2018): “Positive Parenting in Spain: Introduction to the Special Issue", Early Child Development and Care, 188(11), pp. 1503-1513.

ASMUSSEN, K. (2012): The evidence-based parenting practitioner's handbook, Londres, New York, Routledge.

AYUNTAMIENTO DE VITORIA-GASTEIZ (2015): Análisis de los programas y actividades de parentalidad positiva en el Ayuntamiento de Vitoria-Gasteiz [Informe interno del Servicio de Infancia y Familia y la Universidad del País Vasco, UPV/ EHU].

BARUDY, J. y DANTAGNAN, M. (2005): Los buenos tratos a la infancia: Parentalidad, apego y resiliencia, Barcelona, Editorial Gedisa.

BLOOMFIELD, L. y KENDALL, S. (2012): “Parenting selfefficacy, parenting stress and child behaviour before and after a parenting programme", Primary Health Care Research \& Development, 13(4), pp. 364-372. 
BOEKHORST, M.G.B.M.; HULSBOSCH, L.P.; NYKLÍ EK, I.; SPEK, V.; KASTELEIN, A.; BÖGELS, S.; POP, V.J.M. y POTHARST, E.S. (2021): "An online mindful parenting training for mothers raising toddlers: Assessment of acceptability, effectiveness, and personal goals", Mindfulness, 12(2), pp. 519-531.

CONGER, R.D.; MARTIN, M.J. y MASARIK, A.S. (2021): "Dynamic associations among socioeconomic status (SES), parenting investments, and conscientiousness across time and generations", Developmental Psychology, 57(2), pp. 147-163.

CROSNOE, R. (2015): “Continuities and consistencies across home and school systems", en SHERIDAN S., MOORMAN KIM E. (eds.): Processes and Pathways of Family-School Partnerships Across Development, Springer International Publishing, pp. 61-80, <http://doi. org/10.1007/978-3-319-16931-6_4>.

DEHART, T.; PELHAM, B.W. y TENNEN, H. (2006): “What lies beneath: Parenting style and implicit self-esteem", Journal of Experimental Social Psychology, 42(1), 1-17.

FANG, Y.; BOELENS, M.; WINDHORST, D.A.; RAAT, H. y VAN GRIEKEN, A. (2021): "Factors associated with parenting self-efficacy: A systematic review", Journal of Advanced Nursing, pp. 1-21, 〈http:// doi.org/10.1111/jan.14767>.

FIESE, B.H. y FISHER, M. (2019): "Family Context in Early Childhood Education”, en SARACHO, O.N. (Ed.), Handbook of Research on the Education of Young Children, Routledge, pp. 284-30.

GALENDE, N.; SÁNCHEZ DE MIGUEL, M. y ARRANZ, E. (2011): "The role of physical context, verbal skills, Non-parental care, social support, and type of parental discipline in the development of ToM capacity in Five-Year-Old children", Social Development, 20(4), pp. 845-861.

GLYNN, L.M.; DAVIS, E.P.; LUBY, J.L.; BARAM, T.Z. y SANDMAN, C.A. (2021): "A predictable home environment may protect child mental health during the COVID-19 pandemic", Neurobiology of Stress, 14, n- 100291.

GOBIERNO VASCO (2012): Estudio de campo de los programas y actividades de parentalidad positiva existentes en la Comunidad Autónoma del País Vasco, Dirección de Política Familiar y Desarrollo Comunitario/ Consejería de Asuntos Sociales, <https://www.euskadi. eus/contenidos/informacion/publicaciones_ observ_infancia/es_publica/adjuntos/ parentalidad_positiva.pdf〉.

(2018): Plan Interinstitucional de Apoyo a las Familias de la CAE para el periodo 2018-2022, Dirección de Política Familiar y Diversidad/ Consejería de Asuntos Sociales, <https://www.euskadi.eus/ contenidos/plan_departamental/20_plandep_ xileg/es_def/adjuntos/IV\%20Plan\%20 Interinstitucional\%20de\%20Apoyo\%20a\%20 las\%2oFamilias\%2ode\%2ola\%20CAE\%20 2018_2022.pdf).

HAEZI-ETXADI (2017): Parentalidad positiva, promoción del desarrollo psicológico saludable desde las prácticas parentales, Barcelona, Fundación Universitaria Iberoamericana.
HANINGTON, L.; HERON, J.; STEIN, A. y RAMCHANDANI, P. (2012): "Parental depression and child outcomes-is marital conflict the missing link?", Child: Care, Health and Development, 38(4), pp. 520-529.

HUERTA, M.D.C.; ADEMA, W.; BAXTER, J.; HAN, W.; LAUSTEN, M.; LEE, R. y WALDFOGEL, J. (2014): "Fathers' Leave, Fathers' Involvement and Child Development: Are they Related? Evidence from Four OECD Countries", European Journal of Social Security, 16(4), pp. 308-346.

JAEGER, M.M. (2012): "The extended family and children's educational success", American Sociological Review, 77 (6), pp. 903-922.

JUVINYA-CANAL, D. (2013): "Salutogénesis, nuevas perspectivas para promover la salud", Enfermería Clínica, 23(3), pp. 87-88.

LIDBECK, M. y BOSTRÖM, P.K. (2021): “I believe it’s important for kids to know they have two parents: Parents' experiences of equally shared parental leave in Sweden", Journal of Social and Personal Relationships, 38(1), pp. 413-431.

LUGO-GIL, J. y TAMIS-LEMONDA, C.S. (2008): “Family resources and parenting quality: Links to children's cognitive development across the first 3 years", Child Development, 79(4), pp. 1.065-1.085.

MCPHERSON, K.E.; KERR, S.; MORGAN, A.; MCGEE, E.; CHEATER, F.M.; MCLEAN, J. y EGAN, J. (2013): "The association between family and community social capital and health risk behaviours in young people: an integrative review", BMC public health, 13(1), pp. 1-13.

MILTEER, R.M.; GINSBURG, K.R.; COUNCIL ON COMMUNICATIONS AND MEDIA COMMITTEE ON PSYCHOSOCIAL ASPECTS OF CHILD AND FAMILY HEALTH y MULLIGAN, D.A. (2012): “The importance of play in promoting healthy child development and maintaining strong parentchild bond: Focus on children in poverty", Pediatrics, 129(1), pp. 204-213, 〈https://doi. org/10.1542/peds.2011-2953>.

MORGAN, A. y HERNÁN, M. (2013): "Promoción de la salud y del bienestar a través del modelo de activos", Revista Española de Sanidad Penitenciaria, 15(3), pp. 78-86.

NEWBORG, J., STOCK J.R., y WNEK, L. (2011): Inventario de Desarrollo Batelle. Manual de aplicación, Madrid, TEA ediciones.

NIEUWBOER, C.C.; FUKKINK, R.G. y HERMANNS, J.M. (2013): "Online programs as tools to improve parenting: A meta-analytic review", Children and Youth Services Review, 35(11), pp. 1.8231.829 .

ORGANIZACIÓN MUNDIAL DE LA SALUD (2004): Promoting mental health: Concepts, emerging evidence, practice: Summary report, Genova, World Health Organization, <https://www.who.int/ mental_health/evidence/en/promoting_mhh. pdf $>$

PÉREZ-BOTELLA, M.; DOWNE, S.; MAGISTRETTI, C.M.; LINDSTROM, B. y BERG, M. (2015): "The use of salutogenesis theory in empirical studies of maternity care for healthy mothers and babies", Sexual \& Reproductive Healthcare, 
6(1), pp. 33-39.

PESIC, J. y BAUCAL, A. (1996): "Vygotsky and psychoanalysis: Toward dialogue", Journal of Russian \& East European Psychology, 34(1), pp. 33-39.

POURTOIS, J. y DESMET, H. (2006): La educación postmoderna, Madrid, Popular.

RECOMENDACIÓN 19 (2006): Comité de Ministros a los Estados miembros del Consejo de Europa. Políticas de apoyo al ejercicio positivo de la parentalidad. (Adoptada por el Comité de Ministros el 13 de Diciembre de 2006 en la $983^{\circ}$ reunión de los delegados de los ministros), 〈http://www.coe.int/t/dg3/youthfamily〉.

RODRIGO, M.J.; AMORÓS, P.; ARRANZ-FREIJO, E.; HIDALGO GARCÍA, M.; MÁIQUEZ CHAVES, M.L.; MARTÍN, J.C.; MARTÍNEZ, R.A. y OCHAITA, E. (2015): Guía de buenas prácticas en parentalidad positiva. Un recurso para apoyar la práctica profesional con familias, Madrid, Ministerio de Sanidad, FEMP.

RONCALLO, C.P.; BARRETO, F.B. y SÁNCHEZ DE MIGUEL, M. (2018): "Promotion of child development and health from the perinatal period: An approach from positive parenting", Early Child Development and Care, pp. 1.540-1.552, 〈https://doi.org/10.1080/03004430.2018.14 95630>.

ROUBINOV, D.S. y BOYCE, W.T. (2017): "Parenting and SES: relative values or enduring principles?”, Current opinion in psychology, 15, pp. 162-167.

SCHADY, N. (2011): "Parents' education, mothers' vocabulary, and cognitive development in early childhood: Longitudinal evidence from Ecuador", American Journal of public health, 101(12), pp. 2.299-2.307.

SHONKOFF, J.P.; GARNER, A.S.; SIEGEL, B.S.; DOBBINS, M.I.; EARLS, M.F.; MCGUINN, L. y COMMITTEE ON EARLY CHILDHOOD, ADOPTION, AND
DEPENDENT CARE (2012): "The lifelong effects of early childhood adversity and toxic stress", Pediatrics, 129(1), pp. e232-e246.

SERVICIO DE INFANCIA Y FAMILIA y HAEZI-ETXADI (2019): Programa de Parentalidad Positiva del Ayuntamiento de Vitoria-Gasteiz, VitoriaGasteiz, Servicio de publicaciones del Ayuntamiento de Vitoria-Gasteiz.

SHUMOW, L. y LOMAX, R. (2002): “Parental Efficacy: Predictor of Parenting Behavior and Adolescent Outcomes", Parenting Science and Practice, 2, pp. 127-150.

SPAGNOLA, M. y FIESE, B.H. (2007): "Family routines and rituals: A context for development in the lives of young children", Infants \& Young Children, 20(4), pp. 284-299.

SQUIRES, J.; BRICKER, D.D. y TWOMBLY, E. (2009): Ages \& stages questionnaires, Baltimore, Paul $\mathrm{H}$. Brookes, pp. 257-182.

STIGLIC, N. y VINER, R.M. (2019): “Effects of screen-time on the health and well-being of children and adolescents: a systematic review of reviews", BMJ open, 9(1), e023191.

VELASCO, D.; SÁNCHEZ DE MIGUEL, M.; EGURZA, M.; ARRANZ, E.; ARANBARRI, A.; FANO, E. e IBARLUZEA, J. (2014): "Evaluación del contexto familiar en un estudio de salud pública”, Gaceta Sanitaria, 28(5), pp. 356-362.

WAN, M.W.; FITCH-BUNCE, C.; HERON, K. y LESTER, E. (2021): "Infant screen media usage and socialemotional functioning", Infant Behavior and Development, 62, nำ101.509.

WONG, C.W.; TSAI, A.; JONAS, J.B.; OHNO-MATSUI, K.; CHEN, J.; ANG, M. y TING, D.S.W. (2021): “Digital Screen Time During the COVID-19 Pandemic: Risk for a Further Myopia Boom", American journal of ophthalmology, 223, pp. 333-337. 